\title{
Trephine Ostomy: Safe and Simple
}

\section{Aykut Soyder* and Hedef Özgün}

Department of General Surgery, Faculty of Medicine, Adnan Menderes University, Aydın, Turkey

\begin{abstract}
Introduction: Fecal diversion is often indicated in perineal sepsis and palliation of anorectal malignancy. This can be performed without recourse to laparatomy. This study was aimed to assess the outcome of trephine to loop sigmoid colostomy creation.
\end{abstract}

Materials and Methods: Trephine Loop Sigmoid Colostomy (TLSC) creation was evaluated retrospectively in unselected 23 consecutive patients including the data related with patient demographics, ASA grade, anesthetics used and surgical complications.

Results: Between January 2008 and June 2012, 23 patients (17 men, 73.9\% and 6 women, 26.1\%) underwent a TLSC. The mean ages of cases were $58.2 \pm 15.8$ (ranged 34-83). The indications for stoma formation were perineal sepsis in $11(47.8 \%$ ) cases (Figure 2), inoperable anorectal cancer in $7(30.4 \%)$ (Figure 1), recto-vaginal fistula in 2 $(8.7 \%)$, fecal incontinence in $2(8.7 \%)$ and sigmoid volvulus in $1(4.3 \%)$. Nine $(39.1 \%)$ had surgery under regional anesthesia because of being in ASA grade III. One (4.3\%) had laparotomy on the postoperative period due to colostomy descent.

Conclusion: TLSC formation is a minimal invasive method and is a basic and fast fecal diversion technique performed easily in not only those without laparotomy indications, but also in high risk patients with low complication rates.

\section{Keywords: Fecal diversion; Trephine stoma}

\section{Introduction}

In today's surgical implementation, day by day invasive techniques find further usage area on many areas. Similarly, classically, in company with laparatomy, it's possible to make ostomy surgery on the aim of just fecal diversion by Trephine ostomy technique which is safety and basic method without laparotomy [1-3]

The indications for stoma formation without laparotomy are diverse and include; fecal incontinence, complex perianal sepsis, palliation of extensive pelvic malignancy, prior to anal sphincter reconstructive surgery, sigmoid volvulus or neoadjuvant therapy for rectal cancer $[4,5]$.

As a result of it does not need a wide laparatomy incision and a minimal dissection made only in the part of stomach, advantages of this technique are not only less operation time, less postoperative pain, ileus and wound side complication but also less postoperative analgesic requirement, applicability in accordance with regional anesthesia, less duration of hospital state and it provides opportunity earlier to start chemotherapy and radiotherapy if necessary.

Trephine stoma technique is defined by Senapi firstly in 1991 as

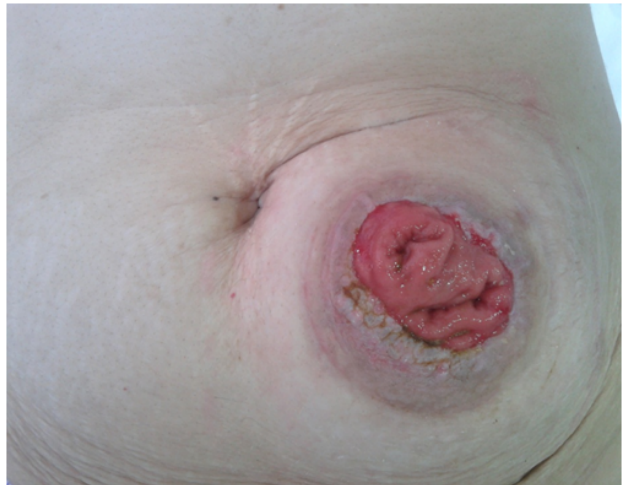

Figure 1: A woman case, aged 67, TLSC applied under regional anesthesia due to inoperable anorectal cancer. an end sigmoid colostomy in 16 cases serie. By the time of literature analyses, it was found that this technique was evaluated only in 6 studies (Table 1).

In this study, indications of the cases TLSC applied advantages of the technique and early period results are evaluated.

We have submitted a short version of this work as a poster during ESS Congress in İstanbul/Turkey.

\section{Patients and Methods}

Between January 2008 and June 2012, cases, decision of colostomy

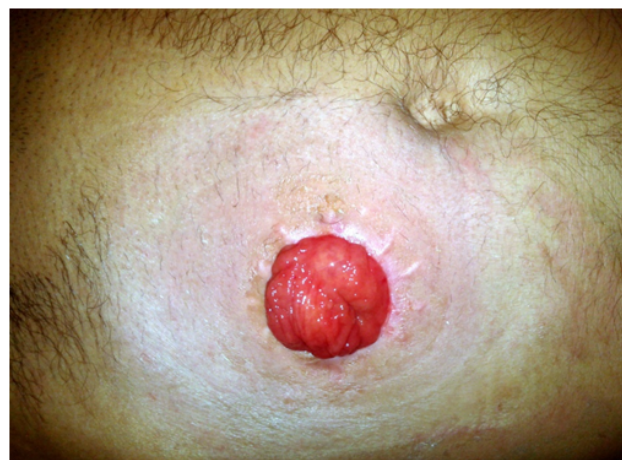

Figure 2: A man case, aged 34, TLSC applied concurrent with definitive treatment with a diagnosis of perineal sepsis.

*Corresponding author: Aykut Soyder, Department of General Surgery, Faculty of Medicine, Adnan Menderes University, Aydın, Turkey, Tel: 90- 256- 4441256 / 1105,90- 532 -3412994; E-mail: asoyder@yahoo.com

Received April 22, 2013; Accepted July 29, 2013; Published August 06, 2013

Citation: Soyder A, Özgün H (2013) Trephine Ostomy: Safe and Simple. Surgery Curr Res 3: 139. doi:10.4172/2161-1076.1000139

Copyright: $\odot 2013$ Soyder A, et al. This is an open-access article distributed unde the terms of the Creative Commons Attribution License, which permits unrestricted use, distribution, and reproduction in any medium, provided the original author and source are credited. 


\begin{tabular}{|c|c|c|c|c|c|c|c|}
\hline Author & $\mathbf{N}$ & Age (years) & Benign/malign & Stay (days) & Conversion & Complications & 30 -days mortality \\
\hline Senapati and Phillips [1] & 16 & 49 & $16 / 0$ & 11 & 1 & 2 late lapatomies & 0 \\
\hline Anderson et al. [2] & 24 & 49 & $20 / 4$ & 9 & $3 / 27$ & 3 stomal related & 0 \\
\hline Nylund et al. [3] & 27 & 51 & $27 / 0$ & $\mathrm{~N} / \mathrm{S}$ & 5 & 1 parastomal herni & 0 \\
\hline Stephenson et al. [5] & 36 & N/S & $\mathrm{N} / \mathrm{S}$ & 10 & 4 & 4 & N/S \\
\hline Patel et al. [7] & 31 & $\mathrm{~N} / \mathrm{S}$ & $5 / 26$ & $\mathrm{~N} / \mathrm{S}$ & 9 & Stomal related & 1 \\
\hline Nessar et al. [8] & 31 & 70 & $13 / 18$ & 9 & 2 & None & 0 \\
\hline
\end{tabular}

N/S: Not Stated

Table 1: Studies related to trephine stoma met in literature.

\begin{tabular}{|c|c|}
\hline No. of patients $(n)$ & 23 \\
\hline Male/Female & 6/17 (\%26.1/73.9) \\
\hline Age (years) ${ }^{\star}$ & $58.2 \pm 15.8(34-83)$ \\
\hline \multicolumn{2}{|l|}{ Indication } \\
\hline $\begin{array}{l}\text { - benign/malignant } \\
\text { - perineal sepsis } \\
\text { - inoperable anorectal cancer } \\
\text { - recto-vaginal fistula } \\
\text { - fecal incontinence } \\
\text { - sigmoid volvulus }\end{array}$ & $\begin{array}{l}16 / 7(\% 69.6 / 30.4) \\
11(\% 47.8) \\
7(\% 30.4) \\
2(\% 8.7) \\
2(\% 8.7) \\
1(\% 4.3)\end{array}$ \\
\hline ASA Grade III (n) & $9(\% 39.1)$ \\
\hline Urgent/Elective & $15 / 8(\% 65.2 / 34.8)$ \\
\hline Anesthetic (GA/Regional) & $14 / 9(\% 60.9 / 39.1)$ \\
\hline Morbidity & 1 (colostomy descent) (\%4.3) \\
\hline Mortality & - \\
\hline
\end{tabular}

*Values given as median \pm standard deviation (min-max), GA: General Anesthesia Table 2: Characteristics of patients undergoing TLSC formation.

taken in our clinic as a result of different indications were evaluated as retrospective, and it was seen that TLSC technique was preferred for 23 cases. These demographic data regarding these 23 cases were evaluated as a descriptive case series in the way of primer pathology colostomy decision taken, ASA value, and anesthesia technique applied and 3 month early period surgical complications (Table 2). Follow up time of TLSC applied cases due to benign primer pathologies (69.6) are limited as 3 months because their colostomies were closed.

\section{TLSC operative technique}

This approach involves performing an operation via a trephine incision through the left iliac fossa. The stoma site is pre-operatively marked. Under anesthetic a standard colostomy opening is made by incising a disk of skin and subcutaneous tissue at the premarked site, usually through the lateral half of the rectus abdominis muscle. The anterior rectus fascia is incised in a cruciate fashion, the rectus muscle is split along its fibers, the posterior sheath is incised, and the peritoneum is opened. The incision may be extended vertically or horizontally if needed. Using a pair of Babcock's forceps the sigmoid colon is grasped and introduced into the wound. Sometimes with a short mesentery or some peritoneal adhesions, lateral mobilization with scissors may be necessary. When the orientation has been confirmed, a loop sigmoid colostomy is then formed without the need for laparotomy.

\section{Results}

By the time data were analyzed belonging to 23 cases TLSC applied, average rate of patients, 17 womens (\%73.9) and 6 mens (\%26.1) was determined as $58.2 \pm 15.8$ (34-83). When loop colostomy was evaluated by the aim of fecal diversion, it was seen that TLSC technique was preferred in 11 cases (\%47.8) perianal sepsis, in 7 cases (\%30.4) inoperable anorectal cancer, in 2 cases (\%8.7) recto-vaginal fistula and fecal incontinence and at 1 case (\%4.3) sigmoid volvulus and it also seemed that loop colostomy was applied because of malign causes totally in 16 benign cases (\%69.6) and 7 benign cases (\%30.4).
In preoperative period, because 9 cases (\%39.1) was evaluated as ASA grade III, regional anesthesia was preferred for all these cases. In other 14 cases (\%60.9) TLSC was applied under general anesthesia. It was determined that this technique was applied at surgical condition to 15 cases (\%65.2) and in other 8 cases (\%34.8) it was applied elective. Apart from laparotomy requirement by the reason of colostomy sink realized in 1 case $(\% 4.3)$ at postoperative $3^{\text {rd }}$ date, there were not any complication and there were no additional morbidity and mortality related to TLSC technique applied at the 3 months early period of cases.

\section{Discussion}

Trephine stoma technique can be applied as ileostomy or colostomy, end or loop. Technique at first was determined at 1991 by Senapati and Phillips [1] as end sigmoid colostomy at 16 cases. In this study, it is reported that this technique was succeeded at 12 cases $(75 \%)$, on the other hand there were complications at 4 cases (25\%) which had laparotomy as a result of insufficient orientation related to sigmoid colon at 1 case, late retraction of stoma related to short mesentery at 2 cases and acute urinary retention development in 1 case. In 1992, Anderson et al. [2] used trephine technique as colostomy or end ileostomy in their study including 24 cases and they described failure at practice in 3 cases (\%12.5), they described prolepsis at 2 cases (\%8.3) during follow up process, and they described parastomal hernia development at 1 case (\%4.2) (Table 1). In 1997, Nylund et al. [3] used loop ileostomy at 7 cases and end sigmoid colostomy at 15 cases via Trephine technique for their study including 27 cases, and it is stated that the technique could not be used as a result of extensive adhesive or immobile sigmoid colon existence at case (\%18.5) [1]. In a similar study, Stephenson et al. [5] emphasized that they used trephine sigmoid colostomy technique $89 \%$ successfully in their study including 36 cases and there were no contraindication depending difference of indication, existence of old laparotomy and obesity. On the other hand, in 1996 Caruso et al. [6] stated that in all 12 cases sigmoid volvulus diagnosed, trephine end colostomy use was succeeded after rectal decompression. In their study, Patel et al. [7] emphasized that 22 of 31 cases, they used Trephine stoma technique successfully, in 9 case (29\%) laparatomy was made as a result of sigmoid colon mobilization difficulty. Similarly in their trephine ileostomy study including 2 cases, Neşşar et al. [8] others emphasized that opening a trephine stoma was a minimal invasive method, and it was a basic and quick fecal diversion technique easily applicable for the cases who had no laparatomy indication.

Besides, laparoscope or endoscope assisted minimal invasive stoma techniques were defined in many study $[2,3]$.

As a common opinion of all these study it is stated that besides trephine stoma use is applicable in extensive indication area, technique is not only applicable but also proof, by comparison with ostomy made via classic laparotomy it has less morbidity and mortality rate, it can be used quickly and so laparotomy is needed rarely.

On the other hand, in literature we could not meet a study evaluates the cases only TLSC used. In comparison to loop ileostomy, for this 
technique, because it does not need exploration to state distal and proximal of bowel loop and complications as ischemia depending malrotation developed by the time; these are seems as additional advantage.

As it seems in the results of this study, it is virtue that it is applicable not only at advanced aged cases who have associated disease under regional anesthesia and elective but also in emergency cases, and it does not require equipments as laparoscope-endoscope.

In conclusion, we consider that TLSC technique had to be evaluated as a proof, quick and easy implementation not only for advanced aged cases having high operation risk, but also for all stoma diagnosed cases laparatomy-free in urgent or elective conditions.

\section{Conflict of Interest}

Trephine loop sigmoid colostomy technique had to be evaluated as a proof quick and easy implementation not only for advanced aged cases having high operation risk, but also for all stoma diagnosed cases laparatomy-free in urgent or elective conditions. Both authors have no conflicts of interest.

\section{References}

1. Senapati A, Phillips RK (1991) The trephine colostomy: a permanent left iliac fossa end colostomy without recourse to laparotomy. Ann R Coll Surg Engl 73: 305-306.

2. Anderson ID, Hill J, Vohra R, Schofield PF, Kiff ES (1992) An improved means of faecal diversion: the trephine stoma. Br J Surg 79: 1080-1081.

3. Nylund G, Oresland T, Hultén L (1997) The trephine stoma: formation of a stoma without laparotomy. Eur J Surg 163: 627-629.

4. Carne PW, Frye JN, Robertson GM, Frizelle FA (2003) Parastomal hernia following minimally invasive stoma formation. ANZ J Surg 73: 843-845.

5. Stephenson ER Jr, Ilahi O, Koltun WA (1997) Stoma creation through the stoma site: a rapid, safe technique. Dis Colon Rectum 40: 112-115.

6. Caruso DM, Kassir AA, Robles RA, Gregory MW, Tsujimura RB, et al. (1996) Use of trephine stoma in sigmoid volvulus. Dis Colon Rectum 39: 1222-1226.

7. Patel P, Wright A, Messersmith R, Palmer J (2001) Does trephine colostomy produce a satisfactory stoma? Colorectal Dis 3: 270-271.

8. Nessar G, Kayaalp C, Bil D, Karadag S (2011) Trephin ileostomy. Turkish Journal of Surgery 27 : $174-5$ 